\title{
Criteria-Based Discharge: Streamlining ENT Patient Discharge In A Large Teaching Hospital
}

M.Hopkins, J Heyman, H El-Hassan, A Farboud, R Mcleod,

D Owens (University Hospital Of Wales, Cardiff)

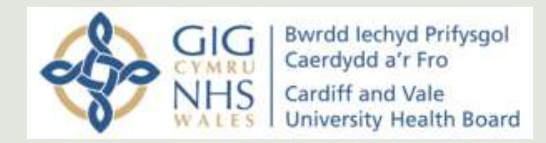

\section{Background}

- $80 \%$ of surgical discharges are considered simple and can be faciliated by nursing staff

- 180 ENT cases/month in day case unit

- Prior to 2015 all cases required ENT senior review prior to discharge resulting in long ward rounds and delays in meeting further clinical commitments or patient discharge

\section{Methods}

- Prospective data collected 9th-10th Jan 2015

- Criteria-based discharge proforma created (see opposite)

- Data on discharges recollected in March-April 2015 and November-December 2017

\section{Results:}

\section{1st Gycle:}

Patient discharge prior to implementation of criteria-based discharge $(n=16)$

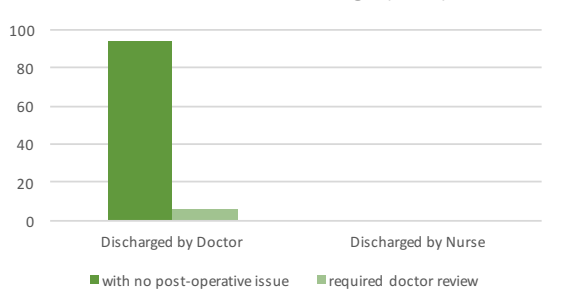

\section{References:}

1. Lees L (2011) Implementing nurse-led discharge. Nursing Times. Vol. 107. No. 39. pp. 18-20.

For further info contact: michael.hopkins2@wales.nhs.uk 2nd Cycle:

Patient discharge using the criteria-based discharge system after implementation of proforma $(n=59)$

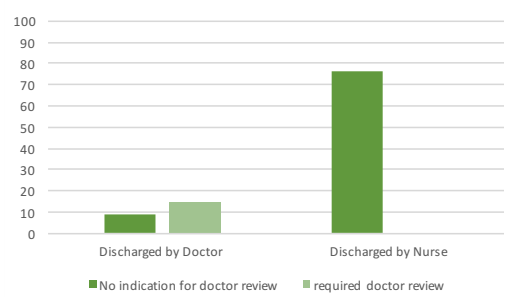

Discussion

- This system has reduced the number of patients requiring review on the ward round by $75 \%$ and maintained this across a 2 year period

- Facilitated timely discharge, resulting in earlier hospital bed space for elective patients, increased patient satisfaction and seniors meeting clinical commitments in a timely fashion.

- Imporved compliance with CBD form completion from 93\%-98\% 